\title{
The CMS tracker upgrade for the High Luminosity LHC
}

\author{
Katja Klein ${ }^{1, *}$ \\ I. Physikalisches Institut B, RWTH Aachen University \\ Sommerfeldstr. 14, 52074 Aachen, Germany \\ E-mail: katja.klein@cern.ch
}

The CMS detector features the world's largest silicon tracker, comprising a strip and a pixel detector. However, the CMS tracker is expected to reach the end of its lifetime after Run 3 of the LHC, and a new device will be installed during Long Shutdown 3. The aim is to at least maintain, and if possible to improve, the performance of the present device, under much harsher conditions in terms of radiation levels, hit rates and pileup. The new tracker will feature an Inner Tracker with silicon pixel modules and an Outer Tracker with strip and macro-pixel silicon modules.

The Inner Tracker will extend the acceptance up to much higher pseudorapidities. More than 4000 hybrid pixel modules with a reduced cell size and with a readout chip based on the RD53 development will be installed. For the Outer Tracker an innovative detector concept was developed, allowing the contribution of tracker data to the first trigger level. For this the data volume that must be sent out at $40 \mathrm{MHz}$ for the trigger decision is reduced already on-module, as each module carries two silicon sensors on top of each other with a distance of a few millimetres, read out by the same front-end ASICs. In that way a rough estimate of the transverse momentum of each particle can be made by exploiting the strong CMS magnetic field of $3.8 \mathrm{~T}$. The module and detector designs are built around this idea.

The detector concept and the expected performance will be presented, and important design choices along with the present status will be discussed.

40th International Conference on High Energy physics - ICHEP2020

July 28 - August 6, 2020

Prague, Czech Republic (virtual meeting)

${ }^{1}$ For the CMS Tracker Group.

* Speaker 


\section{Overview and motivation}

The CMS experiment [1] at the CERN LHC features the world's largest silicon tracker. The tracker is comprised of a pixel detector closest to the beam pipe, and a strip tracker surrounding it. The original tracker was designed for the LHC's design instantaneous luminosity of $1 \times 10^{34} \mathrm{~cm}^{-2} \mathrm{~s}^{-1}$ and an integrated luminosity of $500 \mathrm{fb}^{-1}$. In view of the excellent performance of the LHC, the CMS Collaboration installed a new pixel detector already in 2017, compatible with the increased instantaneous luminosity of $2 \times 10^{34} \mathrm{~cm}^{-2} \mathrm{~s}^{-1}$ [2] reached by the LHC around that time.

The High Luminosity LHC (HL-LHC), scheduled to start operation in 2027, will challenge the LHC detectors with an instantaneous luminosity of $5-7.5 \times 10^{34} \mathrm{~cm}^{-2} \mathrm{~s}^{-1}$, with a pileup (the number of proton-proton collisions per bunch crossing) of 140-200, and with the delivery of $3000-4000 \mathrm{fb}^{-1}$ of data. The CMS Collaboration has decided to exchange completely the tracking system as part of its Phase-2 upgrade [3], with the new tracker to be installed in Long Shutdown 3 (2025-2027).

The main design goals include the capability to deal with the higher expected hit and data rates; an increase in granularity; increased tracking acceptance in the forward region, up to a pseudorapidity of $|\eta| \approx 4$; improved radiation tolerance to cope with fluences of up to $2.3 \times$ $10^{16} \mathrm{n}_{\mathrm{eq}} / \mathrm{cm}^{2}$ and a Total Ionizing Dose of up to $1.2 \mathrm{Grad}$; and the contribution of tracker data to the Level-1 (L1) trigger.

Figure 1 shows the layout of the new tracker. The Inner Tracker (IT) features four barrel layers and 12 disks per side, with in total 3892 silicon pixel modules of two types, comprising $5 \mathrm{~m}^{2}$ of active area and $1.9 \mathrm{G}$ channels. The strip tracker is replaced by the Outer Tracker (OT), which features 5592 modules with macro-pixels and strips (PS modules) in the inner part (at a radius below $60 \mathrm{~cm}$ ), and 7608 modules with strips ( $2 \mathrm{~S}$ modules) further outside. The active area amounts to $190 \mathrm{~m}^{2}$ and the number of channels is $213 \mathrm{M}$. The OT barrel part is made from six layers, with modules in the inner three layers partly arranged in an inclined fashion, while each endcap features five disks.

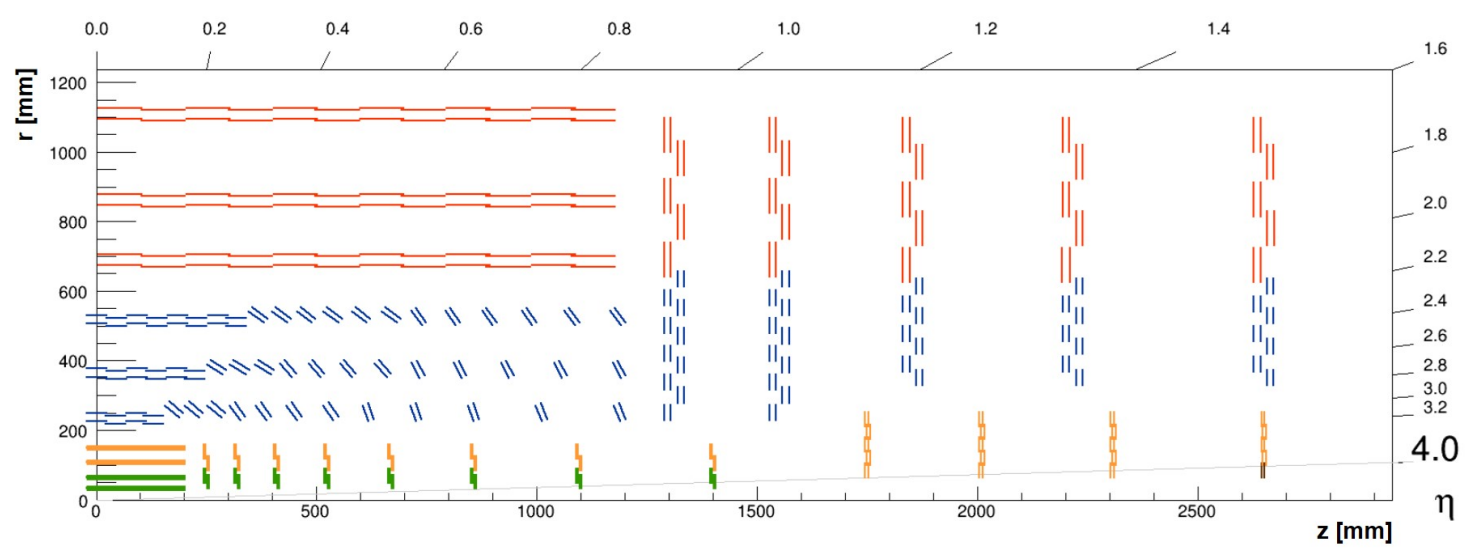

Figure 1: Layout of the CMS tracker, in $r-z$ view, where $z$ points along the beam direction and $r$ is the radial coordinate. One quarter of the tracker is shown. Silicon modules are indicated with coloured lines, where green and orange lines are pixel modules with two or four readout chips, respectively, while blue lines indicate PS modules and red lines indicate $2 \mathrm{~S}$ modules. 

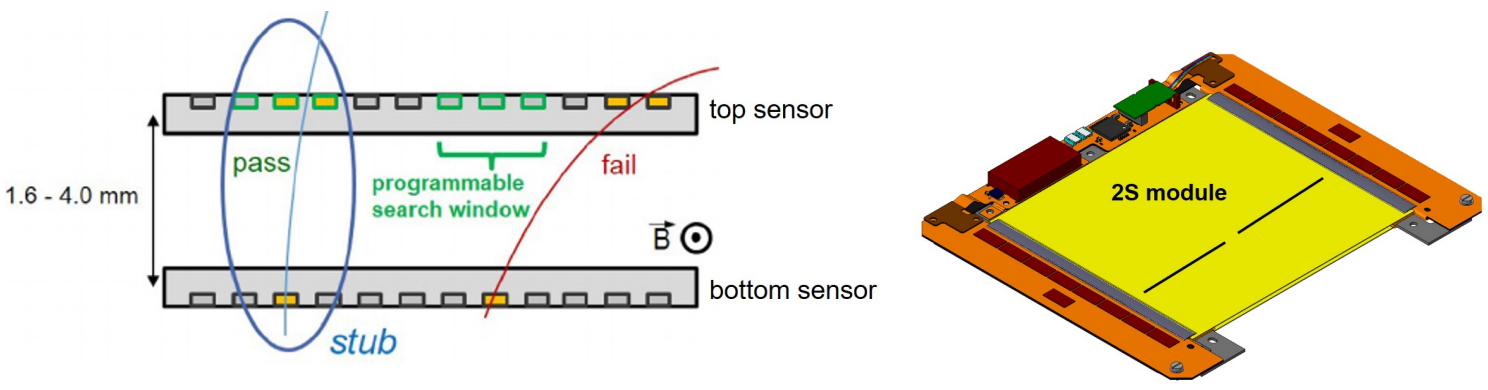

Figure 2: Left: illustration of the identification of the passage of high $p_{\mathrm{T}}$ particles for the $\mathrm{L} 1$ trigger. A cross section through a module is shown, with two sensor planes on top of each other. Orange boxes indicate strips with a hit. In green the search window is indicated. The red track does not pass through its search window due to its low $p_{\mathrm{T}}$. Right: CAD drawing of a $2 \mathrm{~S}$ module. To the left and right of the sensors (yellow) the front-end hybrids are visible, while the service hybrid is visible on the far side. The direction of the strips is indicated with the black lines.

\section{Outer Tracker}

Presently only information from the muon system and the calorimeters is used in the CMS L1 trigger. The use of tracking information would boost the selectiveness and thus performance of the trigger system. However, the complete tracker information cannot be read out at the bunch crossing frequency of $40 \mathrm{MHz}$, due to limitations in bandwidth, and a reduction of the data volume already at the front-end is needed. The vast majority of hits stems from tracks with low transverse momentum, $p_{\mathrm{T}}$, and the key point is to identify tracks with a $p_{\mathrm{T}}$ above a certain threshold. This is achieved by comparing the hit patterns in two closely spaced sensor layers (Fig. 2, left), exploiting the $p_{\mathrm{T}}$ dependent bending of the tracks in the $3.8 \mathrm{~T}$ magnetic field created by the CMS solenoid. The search window in the upper sensor is configurable and together with two (three) different sensor spacings for $2 \mathrm{~S}$ (PS) modules this allows to identify tracks with $p_{\mathrm{T}}>2-3 \mathrm{GeV}$ in all regions of the tracker. The 2-hit tracklets ("stubs") are read out and tracks are formed from them at the back-end. These tracks are passed to the L1 trigger. When a L1 accept signal is received, the complete tracker information is read out at the L1 trigger frequency. This scheme is the design driver for the OT.

The 2S ("strip-strip") module (Fig. 2, right) features two $10 \mathrm{~cm} \times 10 \mathrm{~cm}$ sensors, each with two rows of strips with a cell size of $5 \mathrm{~cm} \times 90 \mu \mathrm{m}$. The strips are wire-bonded to the front-end hybrids (FEHs) that carry each eight CMS Binary Chips (CBC) for readout and one Concentrator Integrated Circuit (CIC) for data aggregation. A service hybrid (SEH) is resonsible for power distribution, data serialization and opto-electrical conversion of the data. The PS ("macro-pixel-strip") module is half as big $(5 \mathrm{~cm} \times 10 \mathrm{~cm})$ and carries a strip sensor with $2.5 \mathrm{~cm} \times 100 \mu \mathrm{m}$ strips on top of a macro-pixel sensor with $1.5 \mathrm{~mm} \times 90 \mu \mathrm{m}$ macro-pixels. The macro-pixel sensor is bump-bonded to 16 Macro Pixel ASIC (MPA) readout chips, while the strips are wire-bonded to the FEHs that each carry eight Short Strip ASICs (SSAs) plus a CIC. The service hybrid is split into two parts, a power hybrid $(\mathrm{POH})$ and a readout hybrid $(\mathrm{ROH})$. Each module is a self-contained unit, connected to the back-end with three wires and two fibers only; common service boards are not used.

Three sensors types are needed (2S, PS-strip, PS-macro-pixels). All designs are final. After extensive R\&D [4] planar n-on-p sensors made of float zone silicon with an active thickness of 

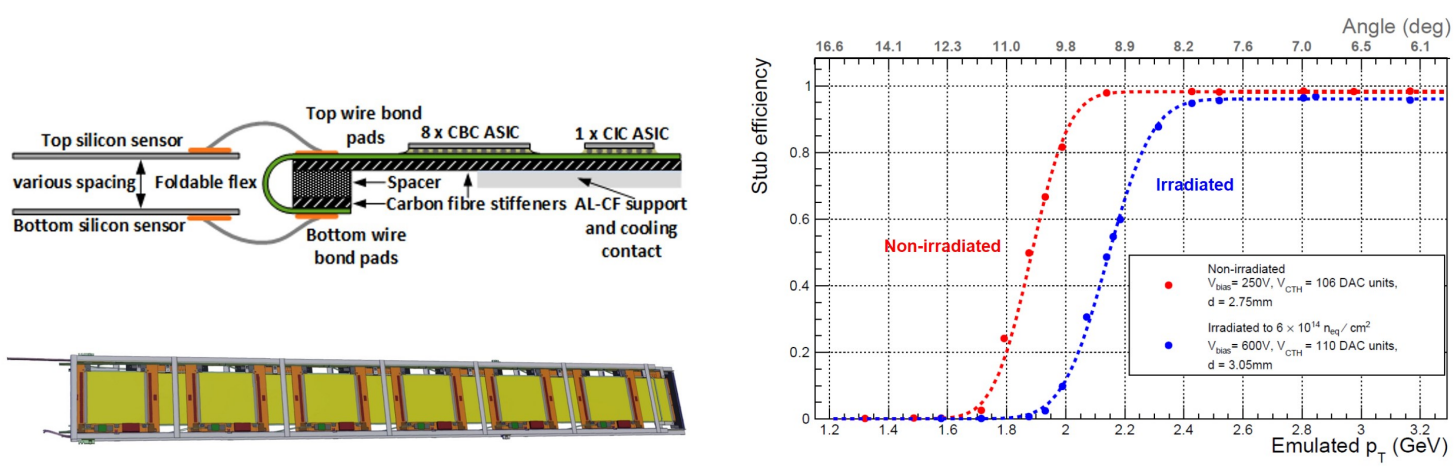

Figure 3: Upper left: schematic cross-section through a $2 \mathrm{~S}$ module, showing how the strips are connected to the FEH and routed through the foldover to the $\mathrm{CBCs}$ on the top side. Right: stub finding efficiency versus emulated $p_{\mathrm{T}}$, for a non-irradiated (red) and an irradiated (blue) module prototype [5]. The turn-on happens at different values due to a different sensor spacing. Lower left: CAD drawing of a $2 \mathrm{~S}$ ladder.

$290 \mu \mathrm{m}$ and a physical thickness of $320 \mu \mathrm{m}$ were chosen. The strips are AC-coupled, while the pixels are DC-coupled. Several irradiation and annealing campaigns with realistic neutron and proton mixes were conducted. For example, the height of the signal on the seed strip after irradiation has been studied as a function of bias voltage and annealing time, and it has been shown that the specification can be met for $2 \mathrm{~S}$ sensors for a bias voltage of $600 \mathrm{~V}$, while for PS sensors a bias voltage of $800 \mathrm{~V}$ is needed in some regions of the tracker after $4000 \mathrm{fb}^{-1}$, which is considered acceptable. All sensors are ordered and the first pre-series batches are presently undergoing Quality Control.

The FEHs are complex 4-layer flexible circuits that are laminated onto carbon fiber (CF) stiffeners and folded on one side around an Aluminium-CF (Al-CF) spacer (Fig. 3, upper left). This allows the readout of the signals of the top and bottom sensors in the same ASIC for the correlation of the hit patterns. This correlation is done in the CBC and MPA for 2S and PS modules, respectively. The CBC is manufactured in $130 \mathrm{~nm}$ technology and features unsparsified binary readout at $320 \mathrm{MHz}$. Each $\mathrm{CBC}$ reads out 127 channels from each sensor, and correlates the hit patterns for stub detection. The CIC is a purely digital chip made in $65 \mathrm{~nm}$ technology. It receives the data from $8 \mathrm{CBCs}$ via 48 lines, and performs formatting and serialization. The data are forwarded to the Low Power Gigabit Transceiver (LpGBT) on the SEH via six lines. On the SEH the LpGBT passes the data at $5 \mathrm{~Gb} / \mathrm{s}$ to the Versatile Transceiver plus (VTRx+), which performs opto-electrical conversion. The SEH carries furthermore two DC-DC converters (the bPOL12V and bPOL2V5) which provide the needed low voltages for the module electronics in a two-step DC-DC conversion powering scheme. While the $\mathrm{CBC}$ is already final (CBC3.1) and in mass production, the CIC2 is under evaluation, and final prototypes of the SEH and FEHs are in production.

The PS module electronics is conceptually very similar, but uses different ASICs (SSA, MPA) and requires twice the bandwidth $(640 \mathrm{MHz}$ and $10 \mathrm{~Gb} / \mathrm{s})$.

About twenty $2 \mathrm{~S}$ modules have so far been built, using prototype components. The full readout chain could be established, and system and beam tests have been performed. It was demonstrated that the module noise is below 1000 electrons, as specified, and that the noise is not increased by using SEH prototypes with DC-DC converters and optical readout, as compared to lab powering 

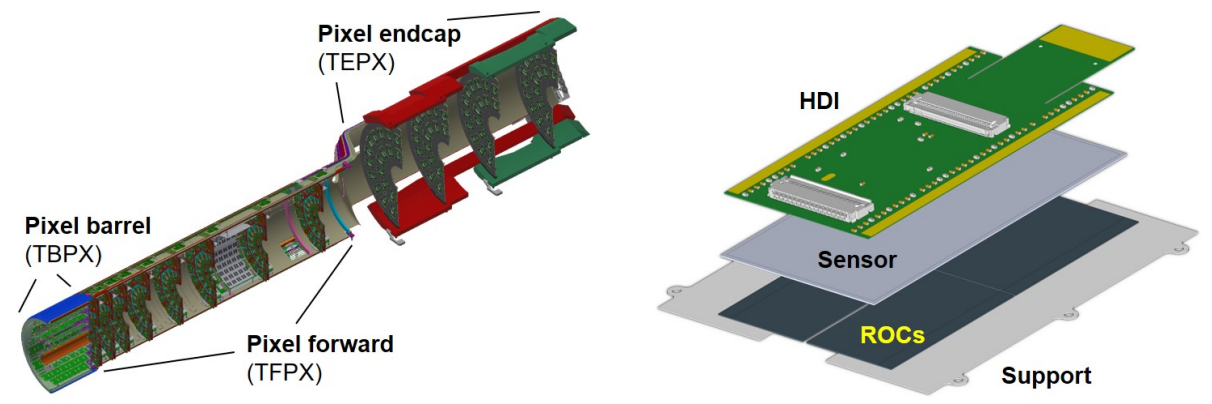

Figure 4: CAD drawing of one quarter of the IT (left) and exploded view of a pixel module (right).

and electrical readout. The stub mechanism was verified in a beam test using non-irradiated and irradiated "mini-modules" with smaller sensors and only two CBCs [5]. Figure 3, right, shows the stub efficiency as a function of emulated $p_{\mathrm{T}}$, where the module was rotated in the beam. A high efficiency of $97-99 \%$ and a $p_{\mathrm{T}}$ resolution of 5-6\% is found both before and after irradiation to twice the fluence expected for those modules.

The modules are mounted on light-weight but stiff CF and/or foam support structures. For example, twelve $2 \mathrm{~S}$ modules are mounted on a "ladder" in the barrel part (Fig. 3, lower left). PS modules are mounted on flat "planks" or rings. In the endcap, modules are mounted on double disks, made of four half disks ("dees"). Two-phase $\mathrm{CO}_{2}$ cooling will be used, with an operational temperature of $-35^{\circ} \mathrm{C}$. This allows the use of thin-walled $(100 \mu \mathrm{m})$ and small diameter $(2 \mathrm{~mm}$ inner diameter) pipes. The module power consumption is $5 \mathrm{~W}$ and $10 \mathrm{~W}$ for $2 \mathrm{~S}$ and PS modules, respectively. Thermal management is thus crucial. The $2 \mathrm{~S}$ modules will be screwed at five points to cooling inserts, while the PS modules will be glued via a phase-change material over the full area to the support structures.

\section{Inner Tracker}

The IT consists of a 4-layer barrel part, a forward part with eight small disks, and an endcap part with four large disks (Fig. 4, left). The detector is constructed such that it can be extracted without removal of the beam pipe, allowing for maintance and potential replacement of modules after e.g. half the expected fluence, given the very large radiation levels that will be present in the inner layers. The hybrid pixel modules (Fig. 4, right) will feature $1 \times 2$ and $2 \times 2$ readout chips (ROCs), bump-bonded to the sensor and glued to support strips. A High Density Interconnect (HDI) glued on top of the sensor is responsible for power and signal distribution. The ROC has to cope with hit rates of up to $3 \mathrm{GHz} / \mathrm{cm}^{2}$. It is based on the $65 \mathrm{~nm}$ ASIC developed within RD53.

In the IT planar sensors will be used, but 3D sensors are still considered an option for the innermost layer. The sensors will be of n-in-p type with an active thickness of $150 \mu \mathrm{m}$. The baseline cell size is $100 \times 25 \mu \mathrm{m}^{2}$, with $50 \times 50 \mu \mathrm{m}^{2}$ also being studied. Details of the pixel geometry (bias dot, implant size) are still being optimized. Extensive studies of the hit efficiency after irradiation have been performed. The hit efficiency is above $99 \%$ for half the expected fluence in layer 1 for bias voltages of about $450 \mathrm{~V}$ and $150 \mathrm{~V}$ for planar and 3D sensors, respectively. A spacial resolution of below $3 \mu \mathrm{m}$ has been measured in test beam experiments. 
The CMS ROC (C-ROC) is developed as a variant of the RD53B chip. Submission is planned for end of 2020. The readout is binary, but time-over-threshold information is also provided. The chip will feature the so-called "linear" analogue front-end, a $432 \times 336$ pixel array, and a Shunt-LDO for serial powering. Serial powering is employed to achieve a low material budget in spite of the front-end power consumption of $50 \mathrm{~kW}$. Up to twelve modules will be connected in a chain, with the ROCs within the module connected in parallel. Chains of three modules (without sensor) have been tested and the noise was similar to that of single modules. The modules will be connected with up to $1.6 \mathrm{~m}$ long electrical links to PCBs (port cards) placed at the pixel detector periphery. Each port card features two LpGBTs and two VTRx+ modules, plus DC-DC converters to power them. An LpGBT receives the data via up to six AC-coupled links at $1.28 \mathrm{~Gb} / \mathrm{s}$.

\section{Expected performance}

The efficiency and fake rate have been studied with simulations using top-quark pair-production events with 200 pileup events superimposed [3]. The performance is comparable to the present tracker at a pileup of 70 , with efficiencies above $90 \%$ in most of the tracker acceptance, and a fake rate of $2-3 \%$. The track parameter resolutions, studied with single muon events, are much better than in the present tracker, with a transverse impact parameter $\left(d_{0}\right)$ resolution of below $10 \mu \mathrm{m}$ and a relative $p_{\mathrm{T}}$ resolution of below $2 \%$ up to $\eta \approx 2.0$ [3]. The performance for the L1 trigger tracks is also excellent, with e.g. a longitudinal impact parameter $\left(z_{0}\right)$ resolution of $1 \mathrm{~mm}$ for top-quark pairs at a pileup of 200 in the center of the detector, profiting from the use of macro-pixels in the PS modules.

\section{Conclusions}

The design requirement to deliver tracking information to the $\mathrm{L} 1$ trigger led to an innovative but challenging detector design. The project is on track for installation in Long Shutdown 3, with first parts of the OT now entering the pre-production phase. The Phase-2 CMS tracker will surpass the performance of the current CMS tracker, under much harsher conditions, and the physics performance of CMS will be boosted by the capability to trigger on tracks at the first trigger level.

\section{References}

[1] CMS Collaboration, The CMS experiment at the CERN LHC, 2008 JINST 3 S08004.

[2] CMS Collaboration, CMS Technical Design Report for the Pixel Detector Upgrade, CERNLHCC-2012-016 (2012).

[3] CMS Collaboration, The Phase-2 Upgrade of the CMS Tracker, CERN-LHCC-2017-009 (2017).

[4] Tracker Group of the CMS Collaboration, Experimental study of different silicon sensor options for the upgrade of the CMS Outer Tracker, 2020 JINST 15 P04017.

[5] Tracker Group of the CMS Collaboration, Beam test performance of prototype silicon detectors for the Outer Tracker for the Phase-2 Upgrade of CMS, 2020 JINST 15 P03014. 